\title{
Método para avaliar a percepção do usuário sobre a qualidade de sistemas de transporte urbano sobre trilhos com utilização da tecnologia neuro-fuzzy
}

\author{
Marcus Vinicius Quintella Cury
}

\begin{abstract}
Resumo: Este trabalho apresenta um método heurístico alternativo, eminentemente qualitativo, baseado na tecnologia neuro-fuzzy, para avaliar e, conseqüentemente, classificar a qualidade e o desempenho de sistemas de transporte urbano sobre trilhos - TUST, segundo a percepção de seus próprios usuários. A tecnologia neuro-fuzzy congrega as principais vantagens da lógica fuzzy e das redes neurais artificiais e foi desenvolvido para funcionar como uma mente coletiva, uma vez que a sua arquitetura hierárquica condensa os graus de avaliação subjetivos atribuídos por usuários. Esse processo ocorre numa combinação de todos os dados em blocos de inferência que utilizam bases de regras fuzzy e expressões lingüísticas, para que a qualidade e o desempenho de um TUST possam ser expressos por meio de uma nota única, numa escala de 0 e 10. Essa nota foi aqui denominada de Grau de Avaliação de Qualidade e de Desempenho - GRADE. Em resumo, o GRADE é a nota atribuída pelos usuários de um TUST, em decorrência da percepção dos serviços ofertados e da performance operacional do sistema, no que diz respeito ao conforto, disponibilidade, regularidade, economia, segurança, rapidez, acessibilidade e relacionamento com o usuário. A tecnologia neuro-fuzzy comprovou ser uma técnica adequada no tratamento do conhecimento heurístico para imitar o raciocínio humano e pareceu ser de fácil entendimento, além de apresentar boa flexibilidade de utilização com dados vagos, incertos e subjetivos.
\end{abstract}

\begin{abstract}
This work presents an alternative heuristic method, eminently qualitative, based on neuro-fuzzy technology, to assessment and consequently to classify the quality and performance of urban rail systems, according to perception of their users. The neuro-fuzzy technology congregates the main advantages of fuzzy logic and artificial neural networks and has been developed to work as a collective mind, since its hierarchical arquiteture adds subjective evaluation degrees which attributes are made by users. This process happens in a combination of all data in inference blocks that uses bases of fuzzy rules and linguistic expressions, so that the quality and the performance of urban rail systems can be express by only one note, in a range between 0 and 10 . This degree is assumed here as Performance and Quality Assessment Degree - GRADE. In short, the GRADE is a note attributed by users of urban rail systems, as a result of perception of offered services and operational performance of system, with respect to comfort, availability, regularity, saving, safety, speed, accessibility and relationship with users. The neuro-fuzzy technology proved to be one technique adjusted in the treatment of heuristical knowledge to imitate the human reasoning and seemed to be of easy agreement, beyond presenting good flexibility of use with vacant, uncertain and subjective data.
\end{abstract}

\section{INTRODUÇÃO}

O transporte urbano sobre trilhos (TUST) é o nome genérico dados aos diversos tipos de transporte ferroviário, tais como os trens urbanos de superfície, os metropolitanos e os sistemas de bondes.

O TUST, assim como os demais modos de transporte, representa um papel fundamental na mobilidade urbana das populações das grandes cidades, cujo desempenho afeta praticamente todas as atividades humanas. O sistema de TUST é constituído por uma tríade - poder público, empresas operadoras e usuários que mantêm uma relação de interdependência, mas com objetivos diferentes entre as partes. Os usuários, por exemplo, desejam o melhor nível de serviço possível, a partir de suas referências de avaliação, como conforto, disponibilidade, regularidade, economia, segurança, rapidez, acessibilidade e relacionamento

Marcus Vinicius Quintella Cury, Departamento de Engenharia de Fortificação e Construção, Instituto Militar de Engenharia. Rio de Janeiro, RJ, Brasil (e-mail: quintella@ime.eb.br).

Manuscrito recebido em 4/4/2007 e aprovado para publicação em $23 / 8 / 2007$. Este artigo é parte de TRANSPORTES, volume $X V$, número 1, de junho de 2007. ISSN: 1415-7713. com a operadora. As empresas operadoras, quando privadas, preocupam-se basicamente com o retorno sobre seus investimentos e, por conseguinte, a criação de valor para seus acionistas. No caso de operadoras públicas, a preocupação deve ficar concentrada no retorno social para os investimentos governamentais. Já o poder público, por sua vez, deve preocupar-se com a correta aplicação do dinheiro do contribuinte e promover a regulação e fiscalização dos serviços concedidos ou sob sua própria responsabilidade.

No presente trabalho, o foco é o usuário, no que diz respeito à sua satisfação em relação a um determinado TUST, em função de suas expectativas como consumidor. Para isso, a empresa operadora, pública ou privada, deve considerar a percepção dos usuários durante sua passagem pelo TUST, com a finalidade de compreender as necessidades e expectativas desses usuários para subsidiar as tomadas de decisão gerencial e operacional da empresa para manter o nível desejado dos serviços prestados.

Os usuários não podem avaliar aspectos técnicos quando estão num sistema de TUST, por isso, devem avaliar somente aspectos qualitativos facilmente perceptíveis e que tenham influência direta na qualidade 
dos serviços prestados pela empresa operadora.

Para dar respaldo legal à participação dos usuários na avaliação dos TUSTs, em 13/02/95, foi promulgada a Lei ${ }^{\circ}$ 8.987, que preconiza, em seu Art. $3^{\circ}$, a cooperação dos usuários na fiscalização das concessões e permissões, realizadas pelo poder concedente responsável. Ainda na mesma lei, o Art. $6^{\circ}$ estabelece que toda concessão ou permissão pressupõe a prestação de serviço adequado ao pleno atendimento dos usuários, conforme estabelecido nas normas pertinentes e no respectivo contrato. A lei entende como serviço adequado aquele que satisfaz as condições de regularidade, continuidade, eficiência, segurança, atualidade, generalidade, cortesia na sua prestação e modicidade das tarifas.

Assim sendo, o método aqui apresentado parte do princípio de que o sucesso de um TUST, sob a ótica do usuário, é uma função de diversos fatores que atuam sobre os próprios usuários. Assim sendo, um método heurístico neuro-fuzzy, arquitetado hierarquicamente, foi desenvolvido, em ambiente participativo, para se obter o grau de avaliação de satisfação do usuário, denominado doravante pela sigla GRADE, numa escala entre 0 e 10. O GRADE é definido como uma medida de intensidade dos impactos que um TUST pode estar causando aos seus usuários. Em outras palavras, o GRADE, baseado no trabalho de Cury (1999), é a nota dada para avaliar um TUST por seus efeitos e ações sobre o bem-estar de seus usuários, atribuída por eles próprios.

Os dados de entrada do método, de ordem eminentemente subjetiva, são "condensados" em um parâmetro único para descrever o GRADE. Já que não existem bons métodos matemáticos para esse tipo de cálculo, partiu-se para um método lingüístico bem definido, tal como a tecnologia neuro-fuzzy, devido à sua habilidade de imitar o processo de decisão humana, conforme preconizado no trabalho de Von Altrock e Krause (1994), adaptado por Cury (1999) na área de transportes e adotado por Goudard (2001) e Fogliatti et al. (2004).

Pode-se considerar que a grande contribuição do presente trabalho é a proposta de adaptação da tecnologia neuro-fuzzy para a avaliação participativa de TUSTs, visto que esta metodologia já vem sendo largamente utilizada no campo da automação industrial, em eletrônica, em sistemas de controle, em diagnósticos médicos e nas avaliações de concessão de crédito bancário.

Em resumo, o método visa elaborar um indicador para avaliar e classificar os TUSTs, sob a ótica do usuário, utilizando a tecnologia neuro-fuzzy, que congrega as principais vantagens da lógica fuzzy e das redes neurais artificiais, ferramentas adequadas para tratar problemas complexos, carregados de impreci- sões e mal formulados sob o ponto de vista das técnicas convencionais.

\section{TECNOLOGIA NEURO-FUZZY}

Zadeh (1965) descreveu a teoria matemática dos conjuntos fuzzy e, por extensão, a lógica fuzzy, apresentando a proposta de fazer uma função de pertinência (ou valores verdadeiros e falsos) operar dentro do intervalo de números reais $[0,1]$.

A lógica fuzzy baseia-se fundamentalmente na crença de Zadeh (1965) de que a incerteza é inerente às ações e acontecimentos da vida humana e é pouco provável que a formulação de modelos matemáticos exatos obtenha sempre sucesso. Assim, Zadeh decidiu observar o mundo e seus problemas de modo que expressões lingüísticas sejam passíveis de operação por computadores, sempre utilizando os graus de pertinência de cada conceito (Meech, 1997).

Na essência, fuzziness é um tipo de imprecisão que deriva de um grupo de elementos dividido em classes que não possuem limites claramente definidos. Tais classes, denominadas de conjuntos fuzzy, surgem, sempre que são traçadas a ambigüidade, a imprecisão, a incerteza e a ambivalência em modelos matemáticos de fenômenos empíricos. Partindo-se do fato que certos aspectos da realidade sempre escapam de tais modelos, a aproximação estritamente binária para o tratamento de fenômenos físicos não é sempre adequada para descrever sistemas no mundo real; e os atributos de sistemas de variáveis freqüentemente emergem de algum tipo de fuzziness. (Veiga, 1994)

As variáveis lingüísticas são importantes na inferência fuzzy. Basicamente, uma variável lingüística é usada para caracterizar aproximadamente tanto os valores das variáveis como as suas relações. Por exemplo, números podem ser utilizados para caracterizar a altura de uma pessoa, mas as palavras podem atribuir categorias a essa altura, tais como "alta", "muito alta", "baixa”, "mediana” etc. A imprecisão introduzida pelo uso das palavras pode ser facultativa ou obrigatória, ou seja, a imprecisão pode ser intencional, com base na ausência de necessidade de maior precisão. Entretanto, freqüentemente a imprecisão é imposta pela falta de meios para se quantificar os atributos de um objeto. Os limites entre os termos lingüísticos são fuzzy, além de existir superposição entre eles. Isso significa que se pode estar mais ou menos certo sobre a verdade ou falsidade de um fato particular (Meech, 1997).

As redes neurais artificiais podem ser definidas como técnicas computacionais que apresentam um modelo matemático inspirado na estrutura neural de organismos inteligentes e que adquirem conhecimento através da experiência. Uma grande rede neural artifi- 
cial pode possuir centenas ou milhares de unidades de processamento; já o cérebro de um mamífero pode ter muitos bilhões de neurônios (Mendes Filho, 1997).

Existe uma estreita relação entre a lógica fuzzy e os sistemas neurais. Um sistema fuzzy tenta encontrar uma região que represente o espaço definido pela interseção, união ou complemento de variáveis fuzzy. Isso é análogo tanto aos modelos de rede neurais quanto aos de programação linear. Entretanto, os sistemas fuzzy aproximam o problema de forma diferente, a partir da mais profunda e robusta epistemologia, ou seja, estudando os limites da faculdade humana de conhecimento e os critérios que condicionam sua validade (Cox, 1992).

A lógica fuzzy apresenta grande potencialidade de aplicação em administração de projetos, formação de preços de produtos, diagnósticos médicos, previsão de vendas, análise de mercado, identificação criminal, orçamento de capital e avaliação de aquisição de empresas, de forma análoga à modelagem aqui proposta, ou seja, quando existe a necessidade da opinião e participação das pessoas envolvidas diretamente no processo. Sayed et al. (2003) afirma que os modelos neuro-fuzzy têm sido muito utilizados por pesquisadores, nos últimos anos, como forma de combinar a representação lingüística dos sistemas fuzzy com as habilidades de aprendizagem das redes neurais artificiais, para diferentes tipos de aplicação, como, por exemplo, modelos de performance comportamental. Outra aplicação interessante de modelos neuro-fuzzy foi pensada por Park (2002) para previsões de curto prazo de volumes de tráfego em rodovias de alta velocidade. Pan (2005) também utiliza uma modelagem fuzzy para avaliar a produtividade e o tempo de construção de rodovias durante os períodos de chuva, com base na análise de séries históricas e na avaliação subjetiva de especialistas, com o objetivo de se estudar o impacto das chuvas no cumprimento do cronograma de obra. No caso da avaliação dos impactos do transporte de cargas em comunidades, Fengxiang et al. (2006) desenvolveram um modelo neuro-fuzzy com base na opinião de especialistas e dados de campo, que resultaram numa análise quantitativa e qualitativa para sub- sidiar tomadas de decisão governamentais.

O problema de um sistema fuzzy é sua dificuldade em tratar muitos fatores, funções de pertinência e/ou regras. As redes neurais já são altamente adequadas para tratar grandes quantidades de dados e classes. Desta forma, as duas técnicas se completam e a fusão de ambas tornou-se uma grande tendência de pesquisa, dando origem, em conseqüência, à tecnologia neuro-fuzzy utilizada no presente trabalho.

\section{ESTRUTURA DO MÉTODO}

O método aqui proposto funciona como uma "mente coletiva”, apresentando uma arquitetura hierárquica que condensa os graus de avaliação atribuídos por usuários, numa combinação de todos os dados em blocos de inferência que utiliza bases de regras fuzzy e expressões lingüísticas, para que a percepção dos usuários em relação ao TUST em estudo possa ser expressa por meio de uma “nota”, ou seja, o GRADE. A estrutura do método, representada no diagrama da Figura 1, baseia-se, fundamentalmente, nas variáveis de entrada (VE), de natureza qualitativa, e possibilita, por meio das técnicas de inferência de um sistema especialista neuro-fuzzy, alcançar o GRADE desejado.

O método utiliza oito VEs e depende da transformação dessas VEs em variáveis lingüísticas, com seus respectivos Graus de Convicção ou de Certeza (GdC), em função da interação entre os usuários do TUST. O método permite a utilização de um número maior de variáveis de entrada, como, por exemplo, as 24 variáveis de entradas que fizeram parte do trabalho original de Cury (1999), sendo 8 quantitativas e 16 qualitativas. Os GdCs são definidos de forma subjetiva, com base mais no pragmatismo do que na estatística. Um grau de certeza de 0,70 , por exemplo, pode ser atribuído por uma pessoa a um parâmetro quando ela tiver certeza que este valor não é o máximo de uma escala de 0 a 1, mas tão somente $70 \%$ dessa.

Em seguida, entra-se no sistema de inferência da rede neuro-fuzzy, arquitetado hierarquicamente, que utiliza bases de regras SE-ENTÃO, proporcionando, assim, um fator de evidência da satisfação dos usuários, por meio de uma variável lingüística final, que,

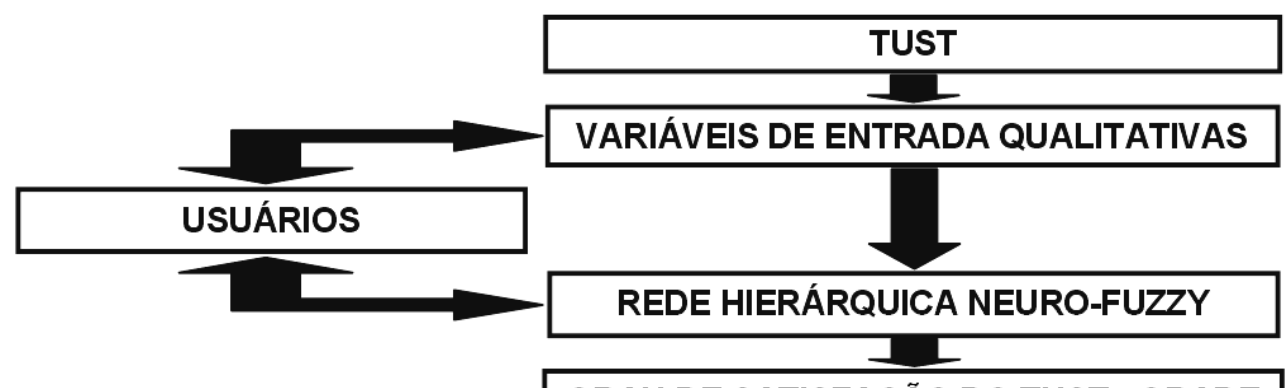

GRAU DE SATISFAÇÃO DO TUST - GRADE

Figura 1. Estrutura do método heurístico 
por um processo de defuzificação lingüística, indica o GRADE do TUST.

A estrutura do método privilegia, em todas as suas fases, a extração da percepção dos usuários do TUST em estudo, ponto fundamental do método heurístico. Segundo o enfoque do presente trabalho, a base natural para a avaliação do desempenho de um TUST é constituída por seus próprios usuários. Desta forma, quando da aplicação do método, deve-se selecionar uma amostra representativa de usuários e, por intermédio de questionários, os usuários atribuem notas, de 0 a 10, para cada VE, segundo sua percepção.

A execução do método, a seleção dos usuários, a elaboração dos questionários para a captação dos sentimentos dos usuários e a interpretação do resultado final são responsabilidades do especialista que gerencia o método aqui proposto.

\section{DEFINIÇÃO DAS VARIÁVEIS DE ENTRADA}

Com base em pesquisas do setor ferroviário (Guedes e Massa, 2005; Transportes, 2000), foram selecionadas as oito VEs qualitativas mostradas na Tabela 1 , que representam os quesitos mais importantes do TUST que influenciam, direta ou indiretamente, os usuários do mesmo.

\section{TRATAMENTO DAS VARIÁVEIS DE ENTRADA}

A falta de medidas para as VEs qualitativas pode ser acomodada com a conversão dos campos de observação em variáveis lingüísticas, por meio da atribuição, por parte dos usuários, de graus de avaliação, numa escala de 0 a 10, com base nos questionários citados anteriormente. Os graus atribuídos pelos usuários são convertidos em expressões lingüísticas, com seus respectivos GdCs, com base nos conjuntos fuzzy e nas regras SE-ENTÃO. Essa fase é denominada fuzificação, uma vez que utiliza os conjuntos fuzzy para as referidas conversões. Na realidade, as VEs qualitativas não precisariam passar pelo processo de fuzificação, pois já são, por excelência, variáveis lingüísticas, cujos termos seriam associados a GdC atribuídos pelos usuários do TUST. Entretanto, no presente método, as VEs qualitativas passam pelo processo de fuzificação, em função da utilização do artifício da atribuição de graus numéricos que refletem os sentimentos dos usuários. Para isso, foram definidos os conjuntos fuzzy genéricos para todas as VEs qualitativas, que apresentam sempre três níveis de termos lingüísticos: um inferior, um médio e outro superior; conforme mostra a Figura 2. A construção desses conjuntos fuzzy tomou por base uma amostra de 30 pessoas, que atribuíram termos lingüísticos para todos os valores da escala de 0 a 10, dentro de um contexto genérico.

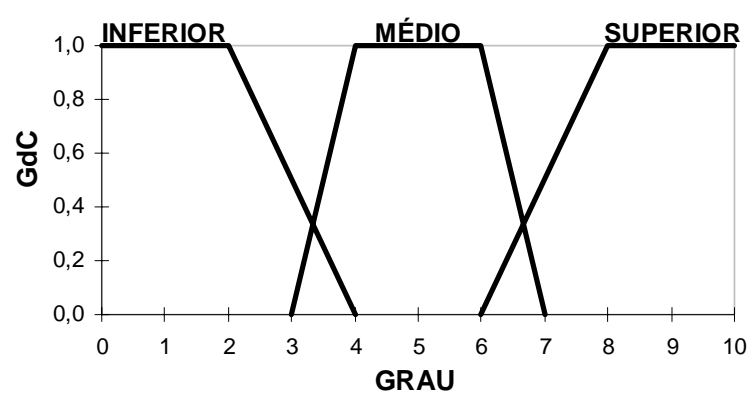

Figura 2. Conjuntos fuzzy genéricos para as VEs qualitativas

Cabe ressaltar que os termos lingüísticos BAIXO, MÉDIO e ALTO, podem ser substituídos, dependendo das características da VE, por quaisquer outros termos equivalentes, tais como RUIM, FRACO, REGULAR, RAZOÁVEL BOM, FORTE, SATISFATÓRIO, EXCELENTE etc, desde que não seja alterado o sentido de graduação do inferior ao superior, mostrado na Figura 2.

Como exemplo, toma-se a variável de entrada REGULARIDADE - REG, cujos termos lingüísticos são BAIXA, REGULAR e BOA, e faz-se a seguinte

Tabela 1. Variáveis de entrada (VE)

\begin{tabular}{|c|c|c|}
\hline Atributos & Sigla & Características \\
\hline Conforto & $\mathrm{CFT}$ & $\begin{array}{l}\text { limpeza dos carros e das estações, comodidade dos assentos, sanitários nas estações, locais de } \\
\text { espera, ar condicionado, lotação dos carros, existência de escadas rolantes }\end{array}$ \\
\hline Disponibilidade & DIS & $\begin{array}{l}\text { capacidade de atendimento a qualquer par de origem-destino, localização das estações, regiões } \\
\text { atendidas }\end{array}$ \\
\hline Regularidade & REG & $\begin{array}{l}\text { cumprimento dos horários disponíveis de saída e dos intervalos entre trens, pontualidade, grau } \\
\text { de certeza nos horários }\end{array}$ \\
\hline Economia & ECO & modicidade tarifária, política de descontos, competitividade com outros modais \\
\hline Segurança & SEG & $\begin{array}{l}\text { segurança pessoal dentro dos trens e das estações e nos entornos, segurança operacional durante } \\
\text { a viagem }\end{array}$ \\
\hline Rapidez & RAP & $\begin{array}{l}\text { tempo de duração de viagem, velocidade operacional durante a viagem, tempo de espera nos } \\
\text { terminais }\end{array}$ \\
\hline Acessibilidade & ACE & $\begin{array}{l}\text { período de operação, integração modal, acessos às estações, plataformas e trens, comunicação } \\
\text { internas nos carros e nas estações }\end{array}$ \\
\hline $\begin{array}{l}\text { Relacionamento com o } \\
\text { usuário }\end{array}$ & REL & $\begin{array}{l}\text { atendimento das bilheterias, presteza dos funcionários, facilidade de comunicação com a gerên- } \\
\text { cia, imagem empresarial }\end{array}$ \\
\hline
\end{tabular}


solicitação a um usuário de um TUST hipotético, após explicar quais as características da variável apresentada: "Numa escala de 0 a 10, que grau você atribuiria para o quesito REGULARIDADE deste TUST?" A seguir, após a resposta do usuário, realiza-se o processo de fuzificação da variável qualitativa REG, conforme mostra a Figura 3. Portanto, entrando-se no gráfico da Figura 2, a variável REG, cujo grau atribuído pelo usuário corresponda a 6,5, é convertida no seguinte vetor da variável lingüística: (BAIXA $=0,00$; REGULAR $=0,40 ; B O A=0,20$ ). Para as demais VEs, o procedimento é o mesmo.

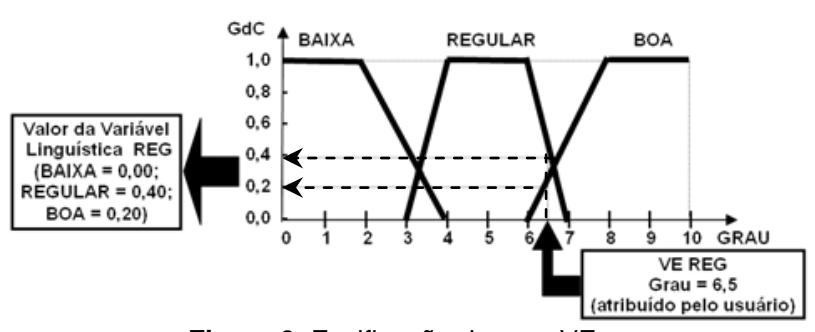

Figura 3. Fuzificação de uma VE

\section{ARQUITETURA DA REDE NEURO-FUZZY}

A arquitetura do método segue o conceito de rede neural hierárquica. Em cada nó da rede dois ou mais elementos são agregados num único elemento, dando origem a um novo nó. Esse novo nó, por sua vez, também se agrega a outros nós, produzidos paralelamente, e dão origem a um novo nó. E assim por diante, até a obtenção do nó final (Cury, 1999).

Com base numa recomendação encontrada no trabalho de Von Altrock e Krause (1994), foi adotada a prática de não se agregar mais que três variáveis em cada nó da rede, para, assim, tornar a inferência mais fácil de ser executada e, no caso da construção da base de regras, permitir a melhor definição dos termos lingüísticos de agregação e de composição das regras, bem como da atribuição dos fatores de certeza de cada regra. Desta forma, no método proposto foram agregados sempre dois elementos em cada nó da rede.

A arquitetura da rede neuro-fuzzy (RNF) possui oito
VEs em sua primeira camada e sempre duas variáveis convergindo para seus nós, num total de sete nós de rede. Cada nó corresponde a uma base de regras fuzzy, denominado de Bloco de Inferência (BI), no qual são computadas as variáveis lingüísticas, por agregação e composição, de modo a produzir um resultado inferido, também na forma de variável lingüística. Assim, nos sete BIs da RNF foram definidas 63 regras.

Em resumo, a arquitetura proposta é composta de sete configurações de sistemas especialistas fuzzy, denominados aqui de Unidades-Padrão de Inferência Fuzzy (UPIF), conforme o esquema da Figura 4, em que duas VEs passam pelo processo de fuzificação e pelo BI, produzindo, em seguida, uma variável de saída (VS), denominada de variável intermediária (VI), caso não corresponda ao último BI da rede. Essa VI, por sua vez, junta-se com outra VI, formando um conjunto de duas novas VEs, configurando, por conseguinte, uma seqüência de UPIFs, até a última camada da rede. Na última camada, a UPIF, também composta por duas VIs, produz a VS definitiva da RNF. Essa VS sofre, então, o processo de defuzificação para que o resultado final seja obtido de forma numérica: o GRADE do TUST em análise (Cury, 1999).

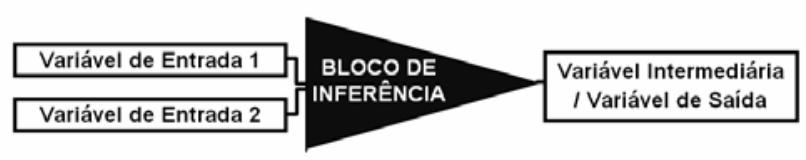

Figura 4. Unidade-padrão de inferência fuzzy (UPIF)

A Figura 5 mostra o desenho esquemático da arquitetura hierárquica do método heurístico neuro-fuzzy proposto, ou seja, da RNF. Os pares de VEs escolhidas para a presente RNF apresentam características correlacionadas, no que diz respeito aos tipos de desempenho estudados, tais como os desempenhos econômico, operacional, humanístico e psicológico. Cabe ressaltar que quaisquer mudanças nas ordens das VEs podem afetar significativamente o resultado final do GRADE, uma vez que haveria uma descaracterização do objetivo proposto. Na verdade, a RNF é genérica e

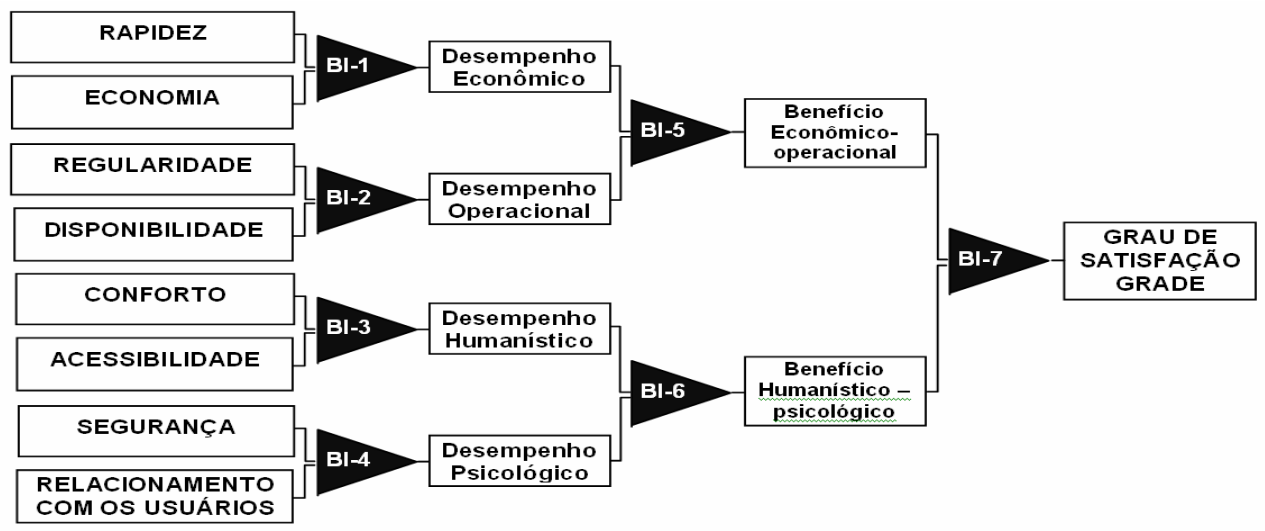

Figura 5. Arquitetura da rede neuro- fuzzy (RNF) 
podem ser escolhidas quaisquer VEs, desde que coerentes entre si, de forma que a RNF possa produzir um resultado final condizente com o objetivo de cada caso estudado.

A arquitetura da RNF deve ser aplicada numerosas vezes, dependendo da quantidade de usuários convocados a participar da execução do método, ou seja, uma $\mathrm{RNF}_{\mathrm{j}}$ para cada $\mathrm{USU}_{\mathrm{j}}$ e, conseqüentemente, $\mathrm{j}$ valores de GRADE como resultados, que, por sua vez, produzirão o $\mathrm{GRADE}_{\text {final, }}$ como será mostrado adiante.

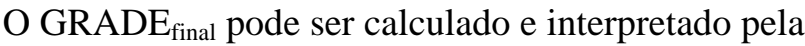
média dos GRADE $\mathrm{j}_{\mathrm{j}}$, segundo os tradicionais métodos da estatística, ou por outros critérios, como a Escala de Likert (ARTESP, 2002), adotada no presente trabalho. Independentemente do método adotado, recomenda-se que sejam selecionados, ao acaso, em diferentes dias, uma amostra de, no mínimo, 100 usuários, para a produção de um GRADEfinal representativo do usuário-padrão do TUST em estudo (Cury, 1999).

\section{BLOCO DE INFERÊNCIA}

Após a conversão de todas as VEs em suas correspondentes variáveis lingüísticas, com seus respectivos GdCs, os BIs já podem ser operados.

Como já foi mostrado, o método hierárquico neurofuzzy é uma rede simétrica composta de sete UPIFs e o cérebro de cada UPIF é o BI, composto pela base de regras SE-ENTÃO, que por sua vez atua como um sistema especialista fuzzy. A base de regras construída depende da camada anterior das variáveis de entrada, para então gerar as variáveis de saída.

O processo de inferência fuzzy identifica as regras e computa os valores lingüísticos da variável de saída, dentro do BI. Desta forma, utilizando-se os conceitos de computação das regras SE-ENTÃO, com base nos operadores MIN-MAX, obtém-se um vetor lingüístico para as variáveis intermediárias de cada UPIF e para a variável de saída final do método, cujos termos lingüísticos foram previamente definidos pelo método.

Cada regra possui um fator de ponderação individu- al, denominado de Fator de Certeza (FC), compreendido entre 0 e 1 , atribuídos pelos usuários, por amostragem simples, que indica o grau de importância de cada regra na base de regras fuzzy, como recomenda Veiga (1994).

A computação da inferência fuzzy consiste de dois componentes: agregação (computação da parte SE das regras) e composição (computação da parte ENTÃO das regras). Os resultados da agregação correspondem aos GdC da partes SE e, por conseguinte, indicam quão adequadas são as regras para o caso em estudo, devidamente ponderadas pelos seus respectivos FC. Para exemplificar, pode-se utilizar a variável REG, utilizada anteriormente, e a variável DISPONIBILIDADE (DIS), para formar uma UPIF, cuja variável de saída é a VI denominada DESEMPENHO OPERACIONAL (DOP), de acordo com a RNF da Figura 5. O grau atribuído pelo mesmo usuário do TUST hipotético às variáveis REG e DIS foi convertido nos seguintes vetores: REG (BAIXA $=0,00$; REGULAR $=$ 0,40 ; $\mathrm{BOA}=0,20$ ) e DIS (BAIXA $=0,30$; MÉDIA = $0,70$; ALTA $=0,00)$. Em seguida, por meio da base de regras do BI, também hipotética (Tabela 2), executase computação da inferência fuzzy, obtendo-se, por conseguinte, o resultado lingüístico da VI (Tabela 3).

Tabela 2. Base de regras do exemplo hipotético

\begin{tabular}{ccc|cc|cc}
\hline & \multicolumn{5}{c|}{$\boldsymbol{S E}$} & \multicolumn{2}{c}{ ENTÃo } \\
\cline { 2 - 7 } $\boldsymbol{R e g r a}$ & Termo & GdC & Termo & GdC & Termo & $\boldsymbol{F C}_{\boldsymbol{i}}$ \\
\cline { 2 - 7 } 1 & BAIXA & 0,00 & BAIXA & 0,30 & BAIXO & 1,00 \\
2 & REGULAR & 0,40 & BAIXA & 0,30 & BAIXO & 0,80 \\
3 & BOA & 0,20 & BAIXA & 0,30 & MÉDIO & 0,60 \\
4 & BAIXA & 0,00 & MÉDIA & 0,70 & BAIXO & 0,80 \\
5 & REGULAR & 0,40 & MÉDIA & 0,70 & MÉDIO & 1,00 \\
6 & BOA & 0,20 & MÉDIA & 0,70 & ALTO & 0,80 \\
7 & BAIXA & 0,00 & ALTA & 0,00 & MÉDIO & 0,60 \\
8 & REGULAR & 0,40 & ALTA & 0,00 & ALTO & 0,80 \\
9 & BOA & 0,20 & ALTA & 0,00 & ALTO & 1,00 \\
\hline
\end{tabular}

Tabela 3. Inferência fuzzy do exemplo hipotético

\begin{tabular}{|c|}
\hline Agregação \\
\hline Regra 1: FC $_{1}$. MIN $\{$ BAIXA; BAIXA $\}=1,00 . \operatorname{MIN}\{0,00 ; 0,30\} \rightarrow\{$ BAIXO $=0,00\}$ \\
\hline Regra 2: $\mathbf{F C}_{2}$. MIN $\{$ BAIXA; MÉDIA $\}=0,80 . \operatorname{MIN}\{0,00 ; 0,70\} \rightarrow\{$ BAIXO $=0,00\}$ \\
\hline Regra 3: $\mathbf{F C}_{3} . \operatorname{MIN}\{$ BAIXA; ALTA $\}=0,60$. MIN $\{0,00 ; 0,00\} \rightarrow\{$ MÉDIO $=0,00\}$ \\
\hline Regra 4: $\mathbf{F C}_{4} \cdot \operatorname{MIN}\{$ REGULAR; BAIXA $\}=0,80 . \operatorname{MIN}\{0,40 ; 0,30\} \rightarrow\{$ BAIXO $=0,24\}$ \\
\hline Regra 5: FC $_{5}$. MIN $\{$ REGULAR; MÉDIA $\}=1,00$. MIN $\{0,40 ; 0,70\} \rightarrow\{$ MÉDIO $=0,40\}$ \\
\hline Regra 6: FC $_{6}$. MIN $\{$ REGULAR; ALTA $\}=0,80$. MIN $\{0,40 ; 0,00\} \rightarrow\{$ ALTO $=0,00\}$ \\
\hline Regra 7: FC $_{7} . \operatorname{MIN}\{$ BOA; BAIXA $\}=0,60 . \operatorname{MIN}\{0,20 ; 0,30\} \rightarrow\{$ MÉDIO $=0,12\}$ \\
\hline Regra 8: $\mathbf{F C}_{8} . \operatorname{MIN}\{$ BOA; MÉDIA $\}=0,80 . \operatorname{MIN}\{0,20 ; 0,70\} \rightarrow\{$ ALTO $=0,16\}$ \\
\hline Regra 9: $\mathbf{F C}_{9} . \operatorname{MIN}\{$ BOA; ALTA $\}=1,00 . \operatorname{MIN}\{0,020 ; 0,00\} \rightarrow\{$ ALTO $=0,00\}$ \\
\hline Composição \\
\hline VI = MAX \{ BAIXO; MÉDIO; ALTO $\}$ \\
\hline VI $=\{$ BAIXO = 0,24; MÉDIO =0,40; ALTO = 0,16 $\}$ \\
\hline
\end{tabular}




\section{TRATAMENTO DA VARIÁVEL DE SAÍDA}

$A \mathrm{VS}_{\mathrm{i}}$ da $\mathrm{RNF}_{\mathrm{i}}$, de cada usuário $\mathrm{i}$, ou seja, o vetor lingüístico do $\mathrm{GRADE}_{\mathrm{i}}$ precisa passar pelo processo de defuzificação para ser transformado em um número real, entre 0 e 10 , para, assim, representar a nota representativa da satisfação desse usuário do TUST em estudo.

O método aqui proposto utiliza a técnica do Centro dos Máximos (CM), ou Centro de Gravidade (CG), no tratamento da variável de saída final, que é uma das técnicas de defuzificação mais utilizadas para transformar um resultado lingüístico novamente num valor numérico, segundo Von Altrock (1997). A maioria dos sistemas de lógica fuzzy utiliza essa etapa porque o resultado desejado, freqüentemente, precisa ser expresso de forma numérica, em vez de maneira lingüística. A Equação 1 calcula o GRADE do TUST por meio da defuzificação pelo Centro dos Máximos:

$$
\operatorname{GRADE}_{j}=\frac{\sum G d C_{i} \cdot X_{i}}{\sum G d C_{i}}
$$

Em que $\mathrm{GdC}_{\mathrm{i}}$ são os graus de certeza dos termos lingüísticos da variável de saída final e $\mathrm{X}_{\mathrm{i}}$ são os valores da escala definida para o GRADE que correspondem aos máximos dos conjuntos fuzzy que definem a variável de saída final.

Assim, o GRADE é, na realidade, um valor ponderado pelos GdC de cada termo lingüístico da variável lingüística GRADE, ilustrado na Figura 6, que apresenta o desenho dos três conjuntos fuzzy que definem a variável de saída do GRADE do sistema de transporte em análise.

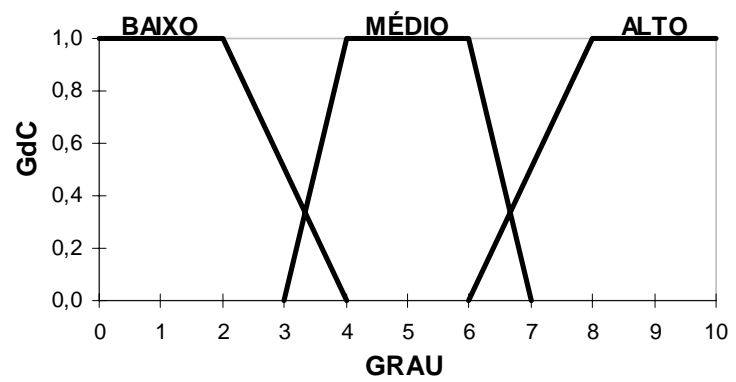

Figura 6. Conjuntos fuzzy da VS - GRADE

O valor do GRADE, entre 0 e 10, representa uma medida de intensidade da satisfação do usuário em relação ao TUST em análise. Para um GRADE igual a 10, a satisfação do usuário pode ser considerada máxima, dentro dos padrões estabelecidos no presente método. Por outro lado, para um GRADE igual a 0, o TUST não satisfaz ao usuário de forma alguma. Já um GRADE intermediário, igual a 7,5, por exemplo, pode ser interpretado segundo critérios a serem estabelecidos, numa escala lingüística qualquer. Um GRADE de 7,5 poderia classificar um TUST dentro de um bom padrão de atendimento ao usuário ou considera-lo como regular para o usuário, dependendo do nível de exigência para o TUST em questão.

Como exemplo, utilizando-se graus atribuídos por certo usuário para um TUST hipotético, entra-se na

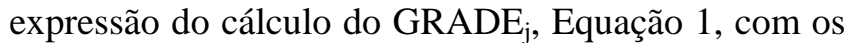
$\mathrm{GdC}_{\mathrm{i}}$ do seguinte vetor lingüístico VS da $\mathrm{RNF}_{\mathrm{i}}$, também hipotético: (BAIXO=0,32; MÉDIO=0,45; $A L T O=0,12)$. Para que seja encontrado o $\mathrm{GRADE}_{\mathrm{j}}$ numérico, encontra-se o valor defuzificado da variável lingüística GRADE $E_{\mathrm{j}}$, numa escala de 0 a 10 , conforme demonstrado na Figura 7. Assim, o GRADE $\mathrm{G}_{\mathrm{j}}$ do exemplo hipotético corresponde a 4,10.

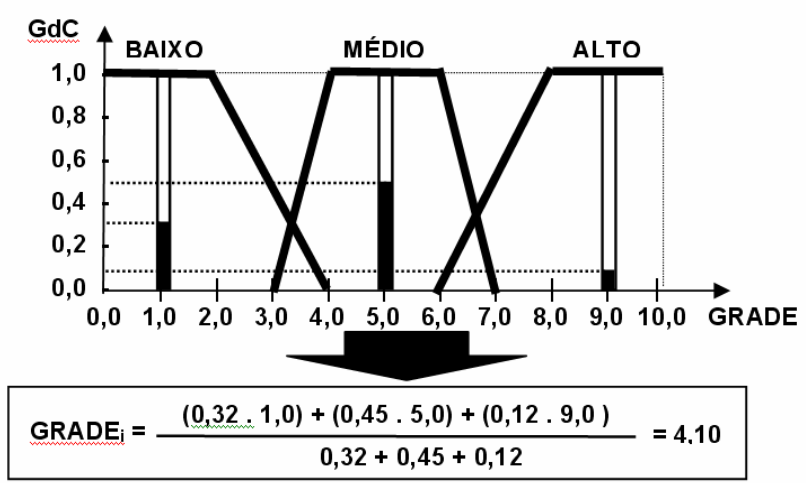

Figura 7. Defuzificação do exemplo hipotético

Da mesma forma, realiza-se este procedimento para cada um dos 100 usuários entrevistados, conforme recomendado anteriormente, e encontram-se 100 valores do GRADE, que serão tratados pela Escala de Likert (ARTESP, 2002), para que seja possível a definição e classificação lingüística de um GRADE único, que represente a percepção do usuário, como um todo, sobre a qualidade e desempenho do TUST.

A Escala de Likert, adaptada pela ARTESP (2002), analisa os 100 valores dos GRADE $E_{\mathrm{i}}$, resultantes das avaliações dos usuários, para encontrar um valor numérico único, entre 0 e 10 , ou seja, o $\mathrm{GRADE}_{\text {final }}$ do TUST em estudo. Em seguida, o valor numérico do GRADE $_{\text {final }}$ deve ser classificado numa escala de cinco expressões lingüísticas - PÉSSIMO, RUIM, REGULAR, BOM e ÓTIMO. Não obstante, a percepção da diferença entre PÉSSIMO e RUIM não é bem definida, pois ambas as expressões refletem um usuário descontente e um TUST precário. Assim sendo, Veiga (2004) recomenda, para cada usuário i, a adoção de outros três termos lingüísticos para enquadrar

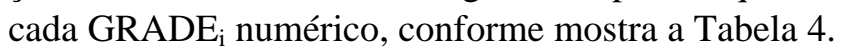

Em resumo, para a definição do $\mathrm{GRADE}_{\text {final }}$ do

Tabela 4. Faixas de classificação dos $\mathrm{GRADE}_{\mathrm{i}}$ numéricos

\begin{tabular}{ccc}
\hline INEFICIENTE & REGULAR & EFICIENTE \\
\hline$[0,3]$ & $(3,7]$ & $(7,0]$ \\
\hline
\end{tabular}


TUST, deve-se adotar o seguinte procedimento, com base no trabalho de Veiga (2004):

- realizam-se, no mínimo, 100 avaliações com usuários do TUST, por meio de 100 RNFs e produzse, por conseguinte, 100 GRADEs numéricos, entre 0 e 10, e seus respectivos termos lingüísticos da Tabela 4;

- com base nos 100 valores de GRADE, contabilizam-se os percentuais de cada termo da Tabela 4 (\%INEFIC, \%REG, \%EFIC), em relação ao total;

- ainda com os 100 valores de GRADEs, calculase a média ponderada, segundo as classes definidas na Tabela 4, chegando-se a um GRADE $E_{\text {final }}$ numérico, pelas freqüências relativas de valores dos GRADEs dos usuários, classificados como INEFICIENTE, REGULAR e EFICIENTE ( $f_{\text {INEFIC, }}$ $f_{R E G}$ e $f_{E F I C}$ ), segundo a Equação 2, cujo valor representa a "nota" do TUST, numa escala de 0 a 10, segundo a "mente coletiva" de seus usuários:

$$
\operatorname{GRADE}_{\text {final }}^{\text {Num. }}=\frac{\left(1,5 \cdot f_{\text {INEFIC }}\right)+\left(5,0 \cdot f_{R E G}\right)+\left(8,5 \cdot f_{E F I C}\right)}{100}(2)
$$

- para definição da classificação lingüística do TUST, faz-se a diferença entre os valores \%EFIC e \%INEFIC, desprezando-se o valor \%REG, chegando-se ao GRADE final $_{\text {lingüístico pela Equação } 3}$ (ARTESP, 2002):

$$
\text { GRADE } \text { fingl }=\% \text { EFIC }-\% I N E F I C
$$

- com o valor do GRADE final Lingüístico da Equação 3, pode-se classificar este valor pela Escala de Likert, conforme mostrado na Tabela 5 (ARTESP, 2002).

Tabela 5. Classificação do $\mathrm{GRADE}_{\text {final }}$ lingüístico pela escala de Likert

\begin{tabular}{cc}
\hline GRADE $_{\text {final }}$ & $\begin{array}{c}\text { Classificação lingüística } \\
\text { do desempenho }\end{array}$ \\
\hline$[+100 \%,+60 \%)$ & ÓTIMO \\
{$[+60 \%,+20 \%)$} & BOM \\
{$[+20 \%,-20 \%]$} & REGULAR \\
$(-20 \%,-60 \%]$ & RUIM \\
$(-60 \%,-100 \%]$ & PÉSSIMO \\
\hline
\end{tabular}

Segundo Veiga (2004), para não ter de fazer uma classificação intermediária na expressão REGULAR, o Índice de Likert tratou de evitar tal necessidade ao não considerar os valores regulares no seu cômputo. Além disso, pode-se observar que a escala da Tabela 5 obedece a um espaçamento uniforme de $40 \%$, definido pela ARTESP (2002).

Como exemplo, seja uma amostra de 100 usuários de um TUST hipotético que produziu os resultados da Tabela 6, após a aplicação do método heurístico neuro-fuzzy aqui proposto. Pode-se notar que o valor numérico de 3,39 para o GRADE $\mathrm{f}_{\text {final }}$ indica uma avalia- ção regular do TUST, muito próximo da ineficiência. Para que haja uma única avaliação, recomenda-se a adoção da Escala de Likert, cujo resultado numérico, para o exemplo dado, pode ser traduzido, segundo a percepção do usuário, como RUIM.

\begin{tabular}{|c|c|c|}
\hline \multicolumn{3}{|c|}{ GRADEs Numéricos para 100 usuários } \\
\hline Intervalo de Classe & $\begin{array}{c}\text { Freqüência } \\
\text { Absoluta }\end{array}$ & $\begin{array}{c}\text { Freqüência } \\
\text { Relativa }\end{array}$ \\
\hline [0 - 3] INEFICIENTE & 57 & $57 \%$ \\
\hline REGULAR & 32 & $32 \%$ \\
\hline EFICIENTE & 11 & $11 \%$ \\
\hline \multicolumn{3}{|c|}{ GRADE $_{\text {final }}$ Numérico = 3,39 } \\
\hline \multicolumn{3}{|c|}{ GRADE $_{\text {final }}=[(1,5 \times 57)+(5,0 \times 32)+(8,5 \times 11)] / 100=3,39$} \\
\hline \multicolumn{3}{|c|}{ GRADE $_{\text {final }}$ Lingüístico = RUIM } \\
\hline \multicolumn{3}{|c|}{$\mathrm{GRADE}_{\text {final }}=\% \mathrm{EFIC}-\% \mathrm{INEFIC}=11 \%-57 \%=-46 \%($ ver Tabela 5$)$} \\
\hline
\end{tabular}

Tabela 6. Exemplo hipotético do cálculo do GRADE final

\section{CONCLUSÕES}

O método proposto traduz o interesse coletivo numa escala numérica, com base em julgamentos subjetivos, trabalhando passo a passo as variáveis qualitativas, sem recair no ponto comum do paradigma atual da monetarização de intangíveis. Outrossim, pode-se concluir que existe um espaço nas políticas públicas de avaliação de sistemas de transporte para a introdução da interatividade entre os interesses privados, a ciência e os cidadãos.

Este trabalho apresentou um método heurístico para o descobrimento de verdades científicas, a partir de regras de decisão que contém informações para a solução desejada. O método heurístico aqui proposto não pode ser considerado como estrito e definitivo, mas coadjuvante e plausível, pois seu principal objetivo é encontrar uma aproximação que pode conduzir a uma solução geral.

A participação dos usuários na aplicação da abordagem heurística do método mostrou-se fundamental para as atribuições de graus para as variáveis de entrada e bases de regras, a fim de garantir uma boa qualidade do GRADE. Desta forma, a tecnologia neurofuzzy comprovou ser uma técnica adequada no tratamento do conhecimento heurístico para imitar o raciocínio humano e pareceu ser de fácil entendimento, além de apresentar boa flexibilidade de utilização com dados vagos, incertos e subjetivos. O método aqui desenvolvido pode também ser aplicado para avaliações de outros modos de transporte, tais como o rodoviário, o hidroviário e o aeroviário, bem como em outros campos, tais como, avaliação de concessões privadas de saneamento, energia e telecomunicações, seleção de recursos humanos, concursos públicos, avaliação de obras públicas, entre outros. 


\section{REFERÊNCIAS BIBLIOGRÁFICAS}

ARTESP (2002) Pesquisa de Satisfação dos Usuários com as Concessões Rodoviárias. Agência Reguladora de Transportes de São Paulo. Disponível em: <http://www.artesp.sp.gov.br>. Acessado em 10 de agosto de 2002.

Cox, E. (1992) The Seven Noble Truths of Fuzzy Logic. Disponível em: $<$ http://www.aptronix.com/fuzzynet>. Acessado em 11 de abril de 1992.

Cury, M. V. Q. (1999) Modelo Heurístico Neuro-Fuzzy para Avaliação Humanística de Projetos de Transporte Urbano. Tese de Doutorado em Engenharia de Produção, COPPE/UFRJ, Rio de Janeiro.

Fengxiang, Q.; Lei, Y. and Lei.X. (2006) Neuro-Fuzzy Logic-Based Model for Assessment of Freight Transportation Implications on Communities. Transportation Research Board 85 th Annual Meeting, Washington, D.C., n. 06-1291, p. 26.

Fogliatti, M. C.; Filippo, S. e Goudard, B. (2004) Avaliação de Impactos Ambientais. Ed, Interciência, Rio de Janeiro.

Goudard, B. (2001) Avaliação Ambiental de Alternativas de Projetos de Transporte Rodoviário com o uso da Lógica Fuzzy. Tese de Mestrado em Engenharia de Transportes, IME, Rio de Janeiro.

Guedes, C. E. F. e Massa, V. L. B. G. (2005), O Sistema de Transporte Coletivo em São Paulo a Partir do Olhar dos Usuários do MetrôSP. Cia do Metropolitano de São Paulo. Disponível em: < http:// www.stm.sp.gov.br/artigos_tecnicos/172.pdf $>$. Acessado em 1 de agosto de 2006.

Meech, J. A. (1997) Fuzzy Logic and Expert Systems. Relatório Interno. University of British of Columbia, Canada.

Mendes Filho, E. F. (1997) Evolutionary Design of MLP Neural Network Architectures. IV Brazilian Symposium on Neural Networks, IEEE Computer Press, Goiânia, Brazil.

Pan, N. F. (2005) Assessment of Productivity and Duration of Highway Construction Activities Subject to Impact of Rain. Expert System with Applications - International Journal, v. 28, n. 2, p. 313-326.

Park, B. (2002) Hybrid Neuro-Fuzzy Application in Short-term Freeway Traffic Volume Forecasting. Transportation Research Record 1802, National Research Council, Washington, D.C., p. 190-196.

Sayed, T.; Tavakolie, A. and Razavi, A. (2003) Comparison of Adaptive Network Based Fuzzy Inference Systems and B-spline NeuroFuzzy Mode Choice Models. Journal of Computing in Civil Engineering, v. 17, p. 123-130.

Transportes (2000) Pesquisa de Avaliação do Nível de Satisfação do Usuário. Ministério dos Transportes. Disponível em: < http://www. transportes.gov.br/Modal/Ferroviario/SU9900.htm>. Acessado em 10 de junho de 2006.

Veiga, M. M. (1994) A Heuristic System for Environmental Risk Assessment of Mercury from Gold Mining Operations. Ph.D. Thesis, The University of British Columbia, Vancouver, Canada.

Veiga, F. J. P. (2004) Técnica Neuro-Fuzzy para a Avaliação do Desempenho Operacional de Rodovias. Tese de Mestrado em Engenharia de Transportes, Instituto Militar de Engenharia, Rio de Janeiro.

Von Altrock, C. and Krause, B. (1994) Multi-Criteria Decision Making in German Automotive Industry Using Fuzzy Logic. Fuzzy Sets and Systems, v. 63, p. 375-380.

Von Altrock, C. (1997) Fuzzy Logic and Neuro-Fuzzy Applications in Business and Finance. Prentice Hall, USA.

Zadeh, L. A. (1965) Fuzzy Sets. Inform and Control, v. 8, p. 338-353. 